This item was submitted to Loughborough's Research Repository by the author.

Items in Figshare are protected by copyright, with all rights reserved, unless otherwise indicated.

\title{
Towards additive manufacture of next generation prosthetics, assessing emerging CAD strategies for improving the existing CAD process order
}

\section{PLEASE CITE THE PUBLISHED VERSION}

http://dx.doi.org/10.1504/IJRAPIDM.2017.10003093

\section{PUBLISHER}

Inderscience

VERSION

AM (Accepted Manuscript)

\section{PUBLISHER STATEMENT}

This work is made available according to the conditions of the Creative Commons Attribution-NonCommercialNoDerivatives 4.0 International (CC BY-NC-ND 4.0) licence. Full details of this licence are available at: https://creativecommons.org/licenses/by-nc-nd/4.0/

\section{LICENCE}

CC BY-NC-ND 4.0

\section{REPOSITORY RECORD}

Bodkin, Troy, Richard Bibb, and Russell A. Harris. 2017. "Towards Additive Manufacture of Next Generation Prosthetics, Assessing Emerging CAD Strategies for Improving the Existing CAD Process Order”. figshare. https://hdl.handle.net/2134/23188. 


\title{
Towards Additive Manufacture of Next Generation Prosthetics, Assessing Emerging CAD Strategies for Improving the Existing CAD Process Order
}

\author{
T. BODKIN ${ }^{1}$, R. BIBB ${ }^{1}$ and R. HARRIS ${ }^{2}$ \\ ${ }^{1}$ Design School, Loughborough University, Loughborough, UK \\ ${ }^{2}$ School of Mechanical Engineering, University of Leeds, Leeds, UK \\ T.Bodkin@lboro.ac.uk, R.J.Bibb@lboro.ac.uk, R.A.Harris@leeds.ac.uk
}

\begin{abstract}
The research project that this work comes from aims to address the issues and inefficiencies of current CAD systems in regards to working on multiple scales, with a particular focus on improving prosthetic design. Working from the micron scale to the macro scale and following on from work that defined a criteria of necessary material properties, this paper is a continuation of previous work that attempts to answer the research question 'How can new CAD strategies be applied to improve the efficiency of producing parts with these necessary material properties?' A selection of emerging CAD strategies from the last five years have been selected with a view of improving the hybrid process order created in the previous study. Each of these processes is introduced, and their pros and cons compared before identifying the areas of the CAD criteria that they can improve efficiency. Testing was performed using the software if it is available to see areas of improvement first-hand.
\end{abstract}

KEYWORDS: Computer Aided Design; Volumetric Modelling; Algorithmic Modelling; Additive Manufacture Format; Surface Modelling

\section{INTRODUCTION}

In an attempt to keep up with the rapid growth of the Additive Manufacturing (AM) field, this research project is attempting to assess the need for improvements in the area of Computer Aided Design (CAD) with a particular focus on enhancing the design and production of the next generation of prosthetics. This paper details the projects second study that endeavours to introduce and test upcoming CAD strategies and asses the opportunities they pose for enhancing current CAD systems. A criteria which defines the necessary CAD features was introduced in the previous study of this project. This design criteria was initially defined by the research group before being adapted for CAD. These criteria are as follows: the model produced must be of a Robust Structure, include Anisotropic properties, be able to represent the scale of Anatomical form, contain information for Multiple Materials and Material Grading, be able to define areas of Porosity for Comfort (permeability), or Lattice Structures to reduce weight where possible and define Cell Channels and Active Surfaces where necessary for cultivating biological interfaces. Each of these criterions takes into account the macro, micro and micron scales needed to accurately represent the necessary details that could be part of a next generation prosthetic limb. 
Previous work available in the conference proceedings of the Fraunhofer Direct Digital Manufacturing Conference 2016 [1], established a control work flow for assessing the CAD criteria. A number of tests were performed using three CAD systems. The chosen systems represented one of the three CAD archetypes, parametric, Non Uniform Rational Basis Spline (NURBS) and polygon mesh. After these tests for each of the criteria were performed, the top ranking system for each step was taken and combined into a hybrid workflow, a flow chart detailing each step that was taken, was produced. The workflow shows how a prosthetic interface with each of the desirable features represented can be made with current CAD. This visual representation of the intellectual load required to produce a socket will aid in identifying the areas of the workflow that require improvements in efficiency. The workflow can be seen in Figure 1. From here the second study commences; following the literature five upcoming $\mathrm{CAD}$ technologies that may improve the efficiency of the modelling process are introduced. The combined result of these two studies will build towards the necessary knowledge for theorising a hypothetical interface for this future CAD system.

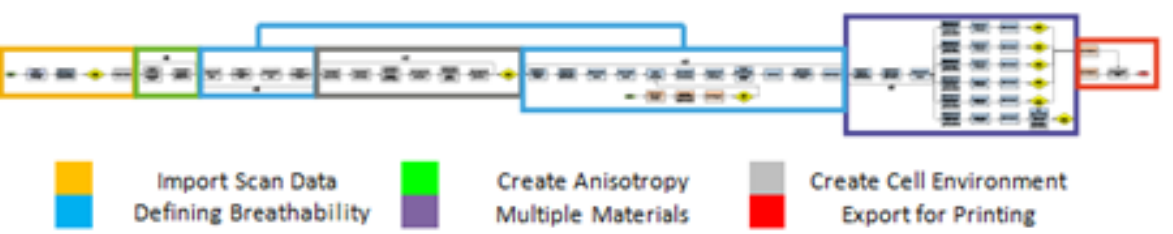

\begin{tabular}{|c|c|c|c|c|c|c|c|c|c|}
\hline Workflow & Criteria & $\begin{array}{c}\text { Import } \\
\text { Geometry }\end{array}$ & $\begin{array}{c}\text { Create } \\
\text { Anisotropy }\end{array}$ & $\begin{array}{c}\text { Cell } \\
\text { Channels }\end{array}$ & Porosity & Latice & $\begin{array}{l}\text { Multiple } \\
\text { materials }\end{array}$ & $\begin{array}{c}\text { Robust } \\
\text { Structure }\end{array}$ & Total \\
\hline \multirow{3}{*}{ Control Model } & Steps & 3 & 2 & 6 & 4 & 14 & $19(3)$ & 3 & 115 \\
\hline & Systems & 2 & 1 & 1 & 1 & 2 & 1 & 2 & 4 \\
\hline & Repetitions & 0 & 6 & 2 & 5 & 2 & 2 & 0 & \\
\hline
\end{tabular}

Figure 1: Breakdown of the control workflow in relation to the CAD criteria, a table of the number of steps and system changes needed to produce a socket, number in brackets is multiplied by the repetitions.

\section{LITERATURE}

To decide on the upcoming systems and plugin's that would be tested in this study a literature review was conducted. Those that showed the most promise for creating the features found in the CAD criteria were selected for further study. Due to the developmental nature of these systems the vast majority of information available comes from online sources, mainly the websites and communities of the software's creators themselves. Where possible, examples of academic literature involving the relevant systems will be given.

The Additive Manufacturing File (AMF) format is an official Americas Society for Testing and Materials (ASTM) standard that describes a framework for a data interchange to address the current and future needs of AM technology [2]. Led primarily by Hod Lipson is an XMLbased format designed to allow any design software to describe the shape and composition of any object to be fabricated on any 3D-printer. The first of AMF's desirable features is its ability to describe volume, material, colour, textures, graded materials and lattices within the metadata of the file type. AMF also has the ability to represent curved geometric triangles, increasing the accuracy of curved surfaces in the mesh, reducing the necessary number of triangles needed to represent the mesh and therefore the file size. AMF is open source, backwards compatible with STL files and boasts drastically reduced file sizes in comparison to STL. Lastly the standard for AMF was published by ASTM in 2012. The major drawback 
of AMF is that it is struggling to find adopters. Hod Lipson (2013) himself states the three reasons he believes that AMF is struggling to find adoption; STL files are not considered bad enough to require a change and the amount of investment in legacy code will keep them relevant for as long as possible. As a consequence of this, equipment manufacturers are reluctant when it comes to implementing AMF support. Lastly the standard for AMF is not freely available to the public, but it is available to all of the equipment manufacturers [3]. At the time of writing not much has changed on the adoption front, but SolidWorks 2015 has included limited AMF support.

Function Representation (or FRep) defines a geometric object by a single real continuous function of point coordinates. Led primarily by Alexander Pasko, the main goal of then project is to develop a rich system of geometric operations and relations [4]. Some of the desirable features of FRep include the combination of algebraic and implicit surfaces, sweeps, volumetric objects and parametric models. FRep can also be used to model point wise object properties such as material, density and colour. FRep claims guaranteed water tight generation of meshes and model integrity for 3D printing, incorporation of advanced microstructures and tiling of geometry at any scale. The one drawback of FRep is that the current commercial application, Symvol, is only available as a plug in for Rhino [5].

T-Splines biggest advantage is that surfaces can contain differing areas of detail. Additional control points are only added where they are needed, typically creating a model with up to 50\% fewer control points than a standard NURBS model. This is achieved by $T$ points, a vertex where one side has an isoparm and the other does not. An isoparm is an iso-parameter curve, a curve along a fixed surface parameter value as explained by Nicholas North (2010) [6].The decrease in control points aids controlling surface smoothness, speeds up surface editing time and ultimately reduces file size. T-splines models can be easily converted back to a NURBS surface model or exported as a mesh and as of 2011 the technology was acquired by Autodesk. A drawback of T-splines is that it requires either Rhino or Autodesk Fusion 360 [7].

The 3D Manufacture Format (3MF) is a new 3D printing format that will allow design applications to send full-fidelity 3D models to a mix of other applications, platforms, services and printers [8]. 3MF is marketed as allowing companies to focus on innovation, rather than on basic interoperability issues, and it is engineered to avoid the problems associated with other 3D file formats. The desirable qualities of 3MF include the ability to fully describe a model whilst retaining internal information such as colour, materials, textures and various other characteristics [9]. 3MF will be open source, compatible with STL and OBJ files, is human readable and boasts the potential for smaller file sizes with more data. Some founders of the 3MF format include major software and hardware developers such as Microsoft, Autodesk, HP and Shapeways. The 3MF format was very early in development at the time of writing, and may struggle to find support from manufacturers and the $3 \mathrm{D}$ printing community [10].

Grasshopper is a free Rhino 3D plug in that works with generative algorithms to allow users to explore and create new complex shapes. The use of generative algorithms provides a new level of accessibility to CAD, should the user know the name of the function that they desire to use. The user interface is visual, dragging in blocks that represent processes and wiring them together. Once a user creates a Grasshopper algorithm it can be used repeatedly with different model inputs, by changing the surface or curve selection for example. Once again Grasshopper is a Rhino plug in, so it requires a copy of Rhino 3D to use [11].

\section{METHODOLOGY}

3.1 Opportunities for Improving the Process Order 
AMF could possibly improve the Robust Structure section of the process order. The creators boast a decrease in file size over STL, which is desirable. Alongside this the incorporation of creating curved triangles to improve the geometric mesh representation of models is also appealing. In regards to Multiple Materials and Material Grading, material choice can be defined within an AMF file, whether it be solid or graded materials. Having the option to define this metadata in the file could greatly reduce the process order length for this section. An expansion of the multi material option is to combine a material with no material, creating an option for Lattice Structures. The empty space can be defined via a voxel bitmap or mesh and then tiled throughout the model allowing for Porosity to be incorporated into structures while saving on file space.

FRep's claim of 'Guaranteed watertight meshes for 3D printing' could also improve the Robust Structure section of the hybrid process order. The pointwise object property generation of FRep will eventually allow users to specify Multiple Materials within the model, and the creators are currently exploring the possibility of functionally Graded Materials via a blend of two volumetric objects with different material properties. Lattice Structure, Porosity for Comfort and Cell Channels could all be improved by FRep and its ability to seamlessly represent geometry on any scale. Being able to make micron level features on a meter scale model will also be very advantageous when addressing the issues of scale.

One issue encountered when defining the hybrid process order was that the Anatomical Form model was constructed of a large number of surface patches when imported into Rhino. The application of T splines could serve to reduce these surfaces, creating a more workable model. The reduction of control points in a T Splines model also affects the file size, reducing it which could aid Robust Structure.

Another file type offering reduced file size over STL, 3MF is desirable for improving the Robust Structure criterion. 3MF claims to allow material information to be included as metadata within the file, showing potential for improving Multiple Materials but at the time of writing details on this new file type are scarce.

Procedural generation of Lattice Structure is one of the more desirable results garnered from the previous study. Grasshopper's algorithmic nature should allow for a method of generating lattice structures with very little intellectual load.

\subsection{Testing Available Processes}

As with the previous work, a set of simple tests was conceived for each system to assess how they could enhance the existing CAD process order.

AMF will be subjected to three tests. The first test assessed the claim that AMF files can be much smaller than STL files, the second will look at how an AMF file can represent multiple material parts while the third will focus on assessing how functionally graded material parts can be defined. The models used in these tests were created in Creo.

For Symvol, a test for producing microstructure and assessing compatibility with other CAD systems was conceived. This test combined elements of the further testing for Porosity for Comfort and Lattice Structure with importing existing polygon meshes from Creo into the Symvol environment and attempting to export the finished STL back, proving how the plug in communicates with other systems. 
For testing Grasshopper, an algorithm that procedurally generates a lattice structure pattern was produced with the aim of reducing the high intellectual load that the Lattice Structure section of the CAD workflow showed in previous work presented.

Now that these tests have been completed they will be applied to an anatomical scale model of a residual limb to produce a socket. This socket will then be compared with the control model and control workflow produced in previous work.

\section{RESULTS}

\subsection{AMF Results}

The first test performed on AMF was to compare the file size of a model that had been saved as both an STL and AMF file. The control for this test was generated in Creo resulting in a file size of 4.96 MB. To convert this model to an AMF file, AMF Utilities was used. This is a free piece of software available for download form the AMF wikispace. To convert, an STL that was created in Creo was imported into the AMF Tools editor and the saved as an AMF file. The resulting file size of the AMF file was 1.25 MB. For accuracy this test was repeated on a number of geometries. Using the same steps as a previous study, an anatomically accurate socket model was created in Rhino and saved as an STL with a size of 29.7 MB. The AMF file for this model had a resulting file size of 8.28 MB. The third and final test was on a chess piece with an STL file size of $2.59 \mathrm{MB}$. When converted to an AMF file its size was $586 \mathrm{~KB}$, a similar ratio of size difference to the other two tests. The AMF tools representation of these files can be seen in Figure 2.

The second test to be performed in the AMF editor was producing multiple material models. To prepare for this test the same model used in the previous test was re-opened in Creo and a simple extrusion was used to split the model in two. Each half was then saved as its own independent STL file. To create a multi material AMF file:

- $\quad$ Flie > Import

- Click 'Edit Amf Objects'

- Click Add > user defines name of new object

- Import desired STL's to user defined AMF object

- $\quad$ Edit > Edit Objects

- Select object shells and assign their materials

- Save as AMF object

In the object editor window the meshes that make up the AMF object are independently selectable and here their material can be changed, allowing the user to create a multiple material AMF object. It is also worth noting that the file size of this split object is $1.35 \mathrm{MB}$, still smaller than the STL file produced in the previous test. 


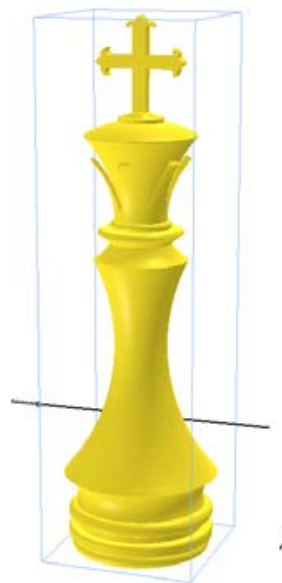

(a)

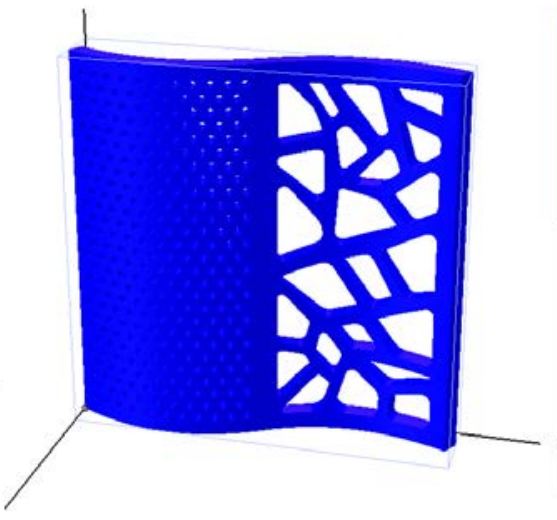

(b)

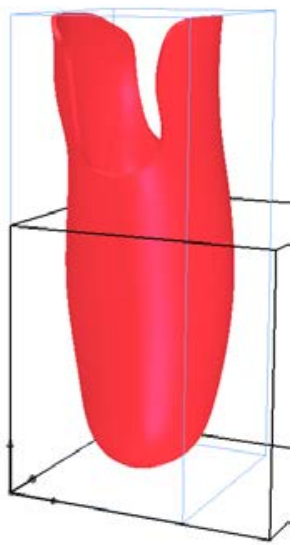

(c)

Figure 2: AMF representations of (a) Chess piece, (b) Previous study test patch (c) Anatomical scale socket

The third test to be performed with AMF editor was an investigation into functionally graded materials. As with the first AMF test the second phase testing model from the previous study was used. Within the Edit Materials window the user has the option of specifying both solid and composite materials. To define the composite material used in this example the code:

Blue: $0.05 *(y+10)$, Yellow: $1-0.05 *(y+10)$

This resulted in a vertical split through the middle of the part with a smooth gradient between the two colour divisions where the materials are blended together.

\subsection{FRep Results}

As this was a test for compatibility with other CAD systems, the initial complex curve shape used in this test was formed in Creo. The shape was then split into an internal shape and an eternal frame both of which were saved as separate STL files. These files were then imported into Rhino.

The Symvol command vMesh allows users to convert volumetric meshes into volume objects that are compatible with further Symvol operations. These volume objects were set aside until they were needed later. A collection of Rhino curves were drawn for use with the vCanal command. This command creates a volume object from a Rhino curve by thickening it to a user defined radius, normal to the curve. The user can also define the number of subdivisions, this effects how smooth the curvature of the canal object will be. The canal object was intended to form the microstructure for the lattice section of the part. vTile arrays the microstructure object within a user defined bounding box. vIntersection was then used to trim the microstructure cube to the size of the internal volume object. The new volume object was then combined with the frame volume object using vBlend. This new volume object was then saved as an STL file for export.

\subsection{Grasshopper Results}

To test Grasshoppers ability to improve Lattice Structure an algorithm for procedurally generating a Voronoi pattern was conceived. The Voronoi pattern was used as the design 
intent for lattice structure in the previous study, a choice inspired by literature and others work on reducing the weight and increasing the aesthetic of prosthetics and orthotics.

As with the second phase testing for Lattice Structure, and due to the fact that Grasshopper has to be used as a plug in for Rhino the complex cured surface that requires a lattice structure is flattened into a 2D surface using the Squish command. From here, Grasshopper is launched using the _Grasshopper command where the interface opens in a separate window. The Voronoi.gh algoritum was created as follows:

- $\quad$ Right click Surface node $>$ Set one Surface $>$ select desired surface in Rhino

- Connect surface node to C pin on Bounding Box node

- Connect BBox to R pin Populate2D node to fill bounding box with points > Add numerical slider to $\mathrm{N}$ pin to control number of points

- Connect Populate 2D node to Voronoi P pin > Connnect BBox to Voronoi B pin

- Wire surface node to Offset Curve node D pin > Right click Offset Curve node $>$ Click expression $>$ User input $-x>$ Wire second numerical slider to D pin

- Connect Voronoi node to pin A on Region Intersect node > Connect OffsetCrv node to pin B on Region Intersect node

- Wire RIntersect node pin R to a second Offset Curve node > Connect the same numerical slider from the first OffsetCrv node to pin $\mathrm{D}$ on this one $>$ repeat expression setup from previous step

- Wire second OffsetCrv C to Fillet Node > create numerical slider and wire it to pin $\mathrm{R}$ on Fillet node

- Adjust numerical sliders to gain users desired pattern

- $\quad$ Right click Fillet node > Bake

It is good practice to turn off the preview option of all nodes except the Surface and Fillet nodes to stop the user interface from becoming cluttered with all the lines that are generated. The sliders can be adjusted to manipulate the pattern and once the user is happy with the appearance of the pattern, right clicking the Fillet node and selecting the Bake option will create the curve network of the pattern in Rhino. At this point Grasshopper can be closed.

The algorithm can be seen in Figure 3. Once an algorithm is created in Grasshopper it can be used for many different applications on various surfaces, reducing how much user involvement is needed to form a lattice structure.

At this point the process continues in the same manner as the second phase of testing in the previous study, the surface is squished back with the generated curves and trimmed by them before the internal surfaces are deleted and the surface is then offset to create a 3D shape.

\subsection{Application}

The available processes were applied to an anatomical form scale model to produce a new socket that has all of the features of the aforementioned CAD criteria.

Grasshopper was used to produce the Lattice Structure by applying the algorithm used in earlier testing. This application had little deviation from what was performed in the previous test. 


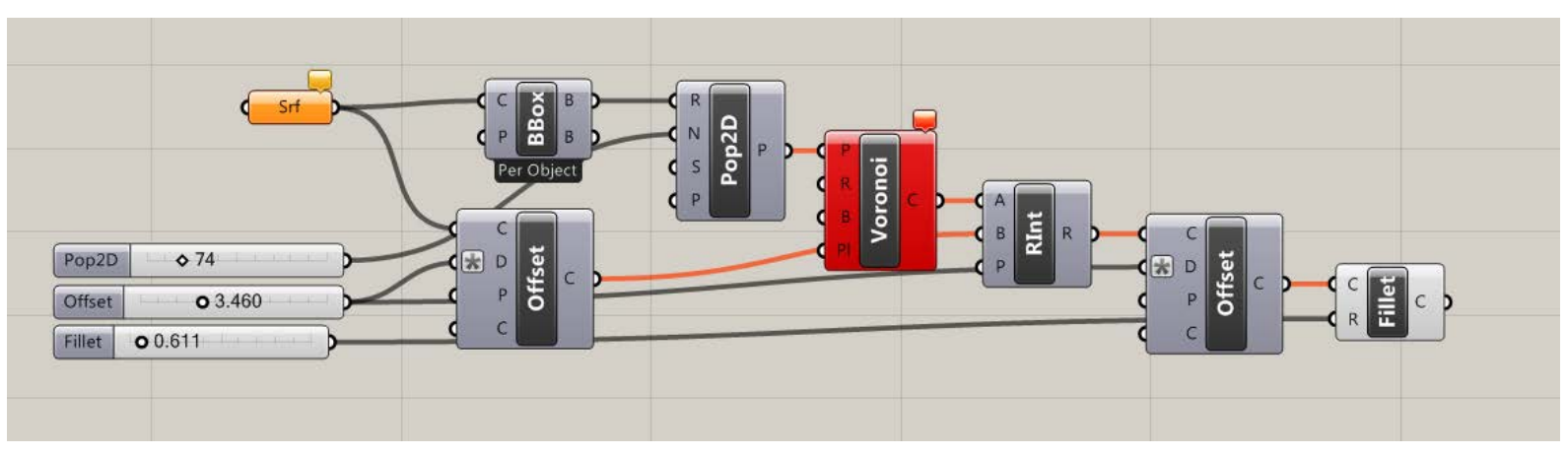

Figure 3: Screen shot of Voronoi.gh Algorithm

Symvol was utilised to produce the porous sections of the socket model. Grasshopper was used to create an offset curve on the surface area where porosity would be applied. This curve was then used to split the surface and once the socket shell was offset these closed regions were converted into meshes with Rhino's_Mesh command. This allowed Symvol's _vMesh command to be used to convert the regions into volume objects. A large block of microstructure, formed from using _vTile command on a .5mm diameter sphere was created to use in the _vIntersection. The resulting volume objects were converted back to meshes to allow work to continue in other CAD systems.

The completed socket model and its material sections were saved as individual STL files for assembly in AMF tools. Each of the sections were assigned their own materials and assembled as a single AMF object. While the individual STL files totalled around 90MB the resulting AMF file came out at $21 \mathrm{MB}$.

The socket model can be found in Figure 4 and the workflow can be found in Figure 5.

\section{CONCLUSION}

AMF performed very well in its tests. The result of the file size test proved the claim of AMF files being smaller than STL files. This was proven with the results of both a direct comparison and a multiple material object producing file sizes nearly one quarter of the same STL file.

As it currently stands, the multiple material step in no more efficient in the creation of a multiple material object as the control process order. The benefit of the approach is that once the AMF object is assembled it is exported as one file with the material information intact, rather than a collection of STL files.

Lastly the material grading test proves that it is possible to represent graded material structures within the AMF file type, but further investigation is required in this area. Being able to define material grading in a point wise fashion would be much more preferable to the current axis based test, as the end goal of defining material properties within a prosthetic socket will require defining multiple points of material grading throughout the 3D space. It is worth noting that with AMF Utilities built in slicer, both multiple material and graded material objects can be exported for Additive Manufacture, provided the machine of choice can read the relevant information in these slice files. 


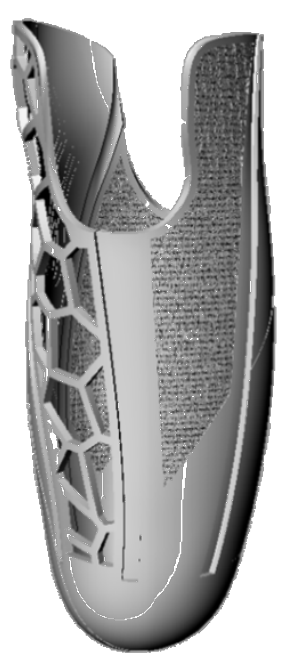

Figure 4: Completed model for a prosthetic socket containing the desirable features defined in the CAD criteria

The resulting STL produced by Symvol was opened in Creo. At some point during the conversion from mesh to volume object, two triangular shaped errors appeared in the frame volume. These errors were assumed to be attributed to the way Symvol visually renders its volume objects, it was not until the object was converted back to an STL that the error was revealed. This is an undesirable aspect of the system and reduced its accuracy when compared to the design intent. The triangulation of the final shape also reduced the accuracy of the STL when compared to the volume object it came from.

The intellectual load required to produce this volume object was very low, improving on all three of the steps in the control tests. The lack of requiring two CAD systems to produce the structure also improves on the control model. The time taken to produce a part like this once one is familiar with the Symvol commands is also impressively short and only 7 steps are required to produce a part with these properties, 2 of which involve import and export.

A huge benefit of Grasshoppers algorithmic based modelling is that it has eliminated the need to transfer between two CAD systems to generate patterns seen in the control test. Another benefit is how once an algorithm has been created it can be opened and used with any Squished surface. This in theory reduces the inefficiency even further as the user would only have to select the surface, adjust the sliders and bake the result to generate a Voronoi pattern for creating a Lattice Structure, vastly reducing the intellectual load. 

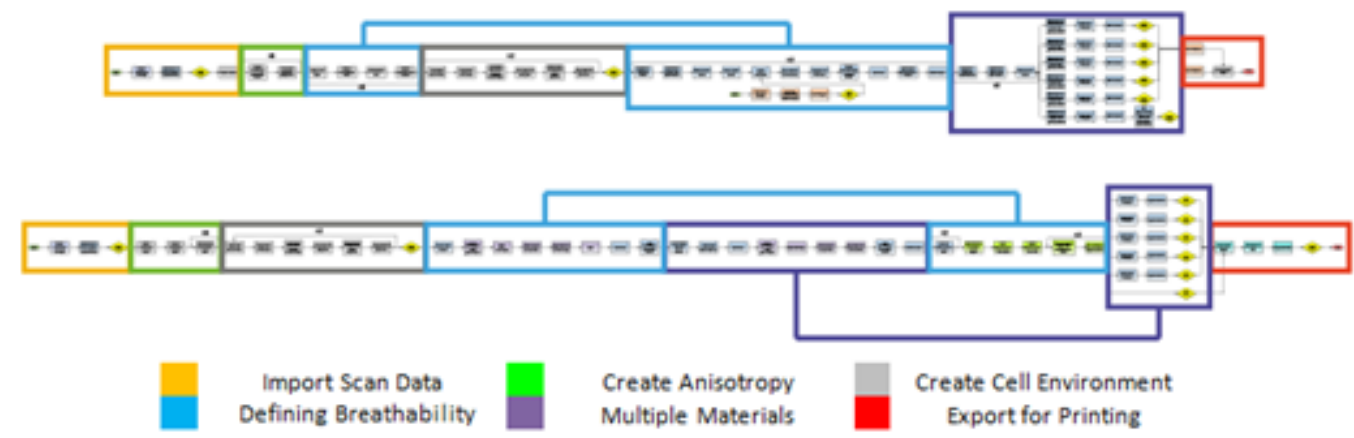

\begin{tabular}{|c|c|c|c|c|c|c|c|c|c|}
\hline Workflow & Criteria & $\begin{array}{c}\text { Import } \\
\text { Geometry }\end{array}$ & \begin{tabular}{|c|} 
Create \\
Anisotropy
\end{tabular} & $\begin{array}{c}\text { Cell } \\
\text { Channels }\end{array}$ & Porosity & Latice & $\begin{array}{l}\text { Multiple } \\
\text { materials }\end{array}$ & $\begin{array}{c}\text { Robust } \\
\text { Structure }\end{array}$ & Total \\
\hline \multirow{3}{*}{ Control Model } & Steps & 3 & 2 & 6 & 4 & 14 & $19(3)$ & 3 & 115 \\
\hline & Systems & 2 & 1 & 1 & 1 & 2 & 1 & 2 & 4 \\
\hline & Repetitions & 0 & 6 & 2 & 5 & 2 & 2 & 0 & \\
\hline \multirow{3}{*}{ Second Study } & Steps & 2 & 3 & 6 & 6 & 12 & 15 & $2(1)$ & 79 \\
\hline & Systems & 1 & 1 & 1 & 1 & 1 & 1 & 1 & 3 \\
\hline & Repetitions & 0 & 0 & 2 & 2 & 2 & 0 & 6 & \\
\hline
\end{tabular}

Figure 5: Comparison between the control workflow found in previous study and the resulting workflow for this study, number in brackets is multiplied by the repetitions.

When comparing the new workflow to the control, a reduction in the intellectual load of producing said model can be seen. The model produced by the new workflow has a greater resemblance to the design intent then that produced by the control. The features produced using these new processes are much closer to the design intent, and also much closer to representing the ideals of said features, such as the microstructure sections being much smaller compared to the arbitrary $2 \mathrm{~mm}$ diameter pores given by the control model.

By reducing the number of steps that need to be repeated, the new workflow consists of 61 steps and 3 system changes, a clear reduction on the control workflow with its 115 steps and 4 system changes. This also served to answer the research question 'How can new CAD strategies be applied to improve the efficiency of producing parts with these necessary material properties?'

While further information is still required for certain aspects of theses upcoming technologies, the knowledge gained from this study will be applied in the next stage of the research project.

The continuation of this work will be to theorise a hypothetical interface for this future CAD system that is built on knowledge of what is possible today and in the near future. 


\section{ACKNOWLEDGEMENTS}

Thanks to Dr Richard Bibb for his continued support and guidance thorough this project and to all the members of the Next-Gen Prosthetics mini CDT for all their input into the project so far.

\section{REFERENCES}

[1] T.Bodkin, R.Bibb, R.Harris, 2016. Towards Additive Manufacture of Next Generation Prosthetics, Specifying a CAD Criteria and Testing it Against the Current State of the Art. In: Fraunhofer Verlag, Fraunhofer Direct Digital Manufacturing Conference DDMC 2016. Berlin, Germany, 16-17 March 2016. Fraunhofer Verlag: Stuttgart .

[2] H.Lipson, ASTM Additive Manufacturing File Format (AMF), 2015, Online, Available at: http://amf.wikispaces.com/

[3] H.Lipson, Why is AMF not yet used for additive manufacturing?, 2013, Online, Available at: https://groups.google.com/forum/\#!topic/stl2/PvhY0X9EHOc

[4] Hyperfun, Shape Modeling and Computer Graphics with Real Functions, 2013, Online, Available at: http://hyperfun.org/wiki/doku.php?id=frep:main

[5] Uformia, Symvol for Rhino, 2015, Online, Available at:http://www.uformia.no/products/symvol-for-rhino/

[6] N.North, Newbie Question - Isoparms?, 2010, Online, Available at: http://www.tsplines.com/forum/viewtopic.php?f=9\&t=29425

[7] Autodesk, Autodesk T splines Plug-in for Rhino, 2013, Online, Available at: http://www.tsplines.com/

[8] 3MF Consortium, Benefits of 3MF for Additive Manufacturing, 2015, Online, Available at: http://3mf.io/

[9] 3MF Consortium, 3D Manufacturing Format Specification and Reference Guide, 2015, Online, Available at:http://3mf.io/wpcontent/uploads/2015/04/3MFcoreSpec_1.0.1.pdf

[10] 3D Inovations, Benefits \& Drawbacks of the New 3MF File Format, 2015, Online, Available at: http://www.3d-innovations.com/blog/benefits-drawbacks-of-the-new3mf-file-format/

[11] S. David, About Grasshopper, 2013, Online, Available at: http://www.grasshopper3d.com/ 